\title{
A rare case of transmigrated mandibular canine and combined orthodontic-surgical management
}

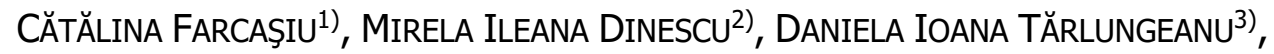 \\ MARILENA BĂTĂIOSU ${ }^{4)}$, CONSTANTIN DĂGUCI ${ }^{5)}$, LUMINIŢA DĂGUCI ${ }^{6)}$, MAGDALENA NATALIA DinA ${ }^{7)}$, \\ OANA-CELLA ANDREI ${ }^{3)}$
}

\author{
1) Department of Pedodontics, Faculty of Dentistry, Carol Davila University of Medicine and Pharmacy, Bucharest, \\ Romania \\ 2) Independent Researcher, Ortholand Clinic, Bucharest, Romania \\ 3) Department of Removable Prosthodontics, Faculty of Dentistry, Carol Davila University of Medicine and Pharmacy, \\ Bucharest, Romania \\ 4) Department of Pedodontics, Faculty of Dentistry, University of Medicine and Pharmacy of Craiova, Romania \\ ${ }^{5)}$ Department of Prevention of Oro-Dental Diseases, Faculty of Dentistry, University of Medicine and Pharmacy of Craiova, \\ Romania \\ 6) Department of Prosthodontics, Faculty of Dentistry, University of Medicine and Pharmacy of Craiova, Romania \\ 7) Department of Dental Techniques, Faculty of Midwifery and Nursing, Carol Davila University of Medicine and Pharmacy, \\ Bucharest, Romania
}

\begin{abstract}
Canines are paramount teeth for esthetics and function. Transmigration of the lower permanent canine is a rare process of alteration of the eruption path toward the opposite part of the mandible. The position of the migrating tooth varies at different ages, so early detection of the tendency to cross the midline is crucial; canines that have already passed through the center of the mandible can be impossible to realign. Transmigration can start any time before the normal age of eruption. Comparing two radiographic images taken at different ages offers important diagnosis information. Camilleri \& Scerri (2003) described a progressive alteration of the tooth position in four steps, from the normal appearance to a low position inside the corpus of the mandible. Following this classification, this article's aim was to present a case of an impacted lower canine that was diagnosed between the second and third stage of transmigration, around the age of 10 , and to detail the combined orthodontic-surgical treatment strategy for "recapturing" and the orthodontic mechanics used to align it. After the examination of cone-beam computed tomography, we have decided to use a lingual arch as a space maintainer and anchorage tool and to surgically expose the tooth. The canine's initial position and the direction of the force of traction used allowed a proper alignment of the tooth inside the dental arch. The periodontal tissues management was important for good healing, stability, and aesthetics. The early detection allowed a proper interdisciplinary orthodontic-surgical management, and a successful outcome.
\end{abstract}

Keywords: transmigration, impacted mandibular permanent canine, lingual arch.

\section{a Introduction}

Tooth impaction is a frequent pathology nowadays, especially due to dental crowding; some teeth just do not find enough space to fit in the maxillary or mandibular arch. In 1974, van der Linden has classified crowding, based on the etiology, in primary, secondary, and tertiary. The first one has a genetic origin and is represented by a tooth size-jaw size discrepancy, the secondary one is produced by environmental factors that shorten the arch length, like interdental caries or premature loss of teeth in the mixed dentition, the last one being a late crowding, determined by aging [1]. Usually, the lower permanent canine erupts after the mandibular first premolar, so alterations in its position or eruption path can occur [2]. In a study evaluating almost 1600 panoramic X-rays, 22 impacted canines were found, most of them being represented by maxillary canines $(0.93 \%)$, while the impaction of the lower canine is rarer, some authors finding it only with a frequency of $0.37 \%$ [3].

Impacted teeth, including the lower canine, tend to migrate in the dental arch [4]. When the canine passes the lower midline, the process is called transmigration [5]. Tarsitano et al. [6] include in this term only the impacted teeth that cross the lower midline, with smaller or bigger amounts from the mesio-distal canine width; other authors, like Joshi [7] and Auluck et al. [8], suggest that the tendency of crossing in the opposing hemimandible is much more important than the actual position of the tooth. Howard suggests that the older the patient, the more dislodged and situated farther away from the midline is the canine, because it has more time to migrate [9].

The eruption process of a canine begins in the mixed dentition, mostly in the early phase [10]. The standard time of eruption for lower canines is between nine and 10 years of age. Chaitanya et al. analyzed the mean age

This is an open-access article distributed under the terms of a Creative Commons Attribution-NonCommercial-ShareAlike 4.0 International Public License, which permits unrestricted use, adaptation, distribution and reproduction in any medium, non-commercially, provided the new creations are licensed under identical terms as the original work and the original work is properly cited. 
for eruption in a sample 1654 school children. For the lower canine, the mean value was around 11.7 years, with different mean values found between the right and left teeth (earlier eruption on the left side) and between girls and boys (earlier eruption for girls) [11]. The mean incidence of transmigrated canines is $0.3 \%$ [12]. Aydin et al. reported an incidence of $0.31 \%$ in a Turkish population, with six maxillary and eight mandibular transmigrations [13]. In another study, the incidence was $0.12 \%$ and occurred only for mandibular canines [3]. Most of the studies found more frequent unilateral transmigrated canines $[7,10,12,14]$, but there are case reports with bilateral transmigration of mandibular cuspids [15-17]. The incidence of transmigrated canines was found higher in females than in males by Joshi [7] and Peck [10], but Joshi presented more frequent transmigration cases on the left side, while Peck recorded an even distribution.

The etiology of transmigration and also its mechanism are still not clear. An explanation for malpositioned or unerupted teeth is the theory of abnormal displacement of the dental lamina during the formation of the embryo [7]. Some authors suggest that eruption is determined by the proper function of the dental follicle, and also by the mechanism of resorption of bone through osteoclasts [18]. Another factor is the radio-transparent enlargement of the follicular space, sometimes resembling a cyst [19]. Other theories of loco-regional causal factors mention cysts, mechanical obstacles like the persistence of the temporary teeth, tumors, odontomas, supernumerary teeth, crowding and an increased length of the canine crown [20].

\section{Aim}

In this paper, our aim was to present a case of a female patient having an impacted lower canine that was diagnosed between the second and third stage of transmigration, around the age of 10 , and to describe in detail the combined orthodontic-surgical treatment strategy for "recapturing" the malpositioned tooth and also the orthodontic mechanics used to align it in order to obtain a functional and esthetic result.

\section{ㄷ Case presentation}

A 10-year-old female patient was already being treated in our Clinic (Ortholand Clinic, Bucharest, Romania) for two years. Between the age of 8 to 10 , during periodical check-ups, an abnormal swelling, hard at palpation, appeared in the left mental fold. The patient was in mixed dentition stage with the upper first premolars and the left lower first premolar fully erupted, and three temporary canines exfoliated, except for tooth number 7.3, which was still present and lacking mobility. Additional records were made, consisting of intraoral and extraoral digital images (Figure 1, a-c), and a left hemi-mandibular cone-beam computed tomography (CBCT).

The dentition was in a good condition, with the permanent teeth being sealed. The personal hygiene, though, was insufficient and determined the presence of gingivitis, and also bleeding on probing. There were given new instructions about the proper brushing technique and regular check-ups were performed to improve the periodontal health before starting the treatment. The occlusal relationship was class III at the level of both molars and canines. The dental midlines did not coincide, the upper one being moved to the right. The overjet and overbite were in normal parameters. There was insufficient space in the upper arch for the permanent cuspids.

Patient had an old panoramic X-ray from the age of eight, so we were able to compare it with the images from the CBCT and we could identify that the left lower permanent canine was impacted, in a process of transmigration. We could also determine the high velocity of the migration process and establish the complexity of the case (Figure 2, a and b). The tooth was in a mesially inclined position, with an angle between the long axis of the tooth and a vertical perpendicular from its apex of $36^{\circ}$.
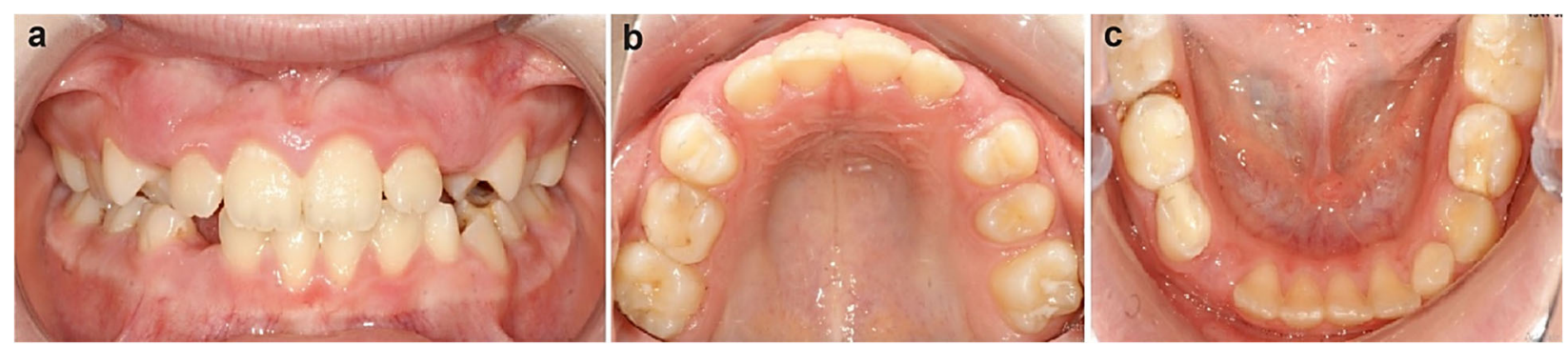

Figure 1 - (a-c) Clinical intraoral examination: frontal view in occlusion, maxillary and mandibular arches in occlusal views.
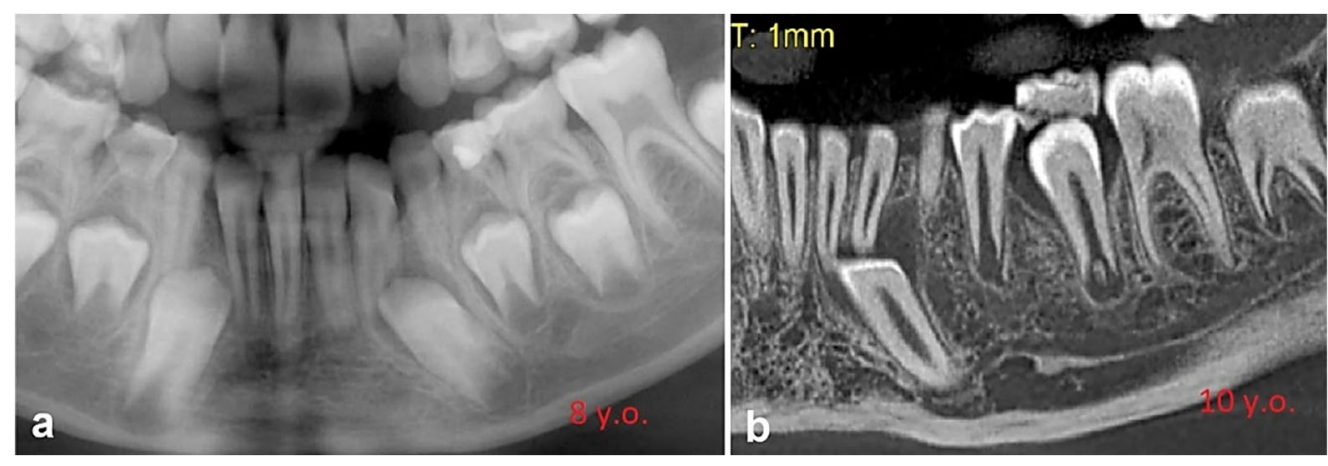

Figure 2 - (a) Detail from the 8-year-old panoramic $X$-ray and (b) the 10-year-old initial pretreatment section from $\mathrm{CBCT}$. CBCT: Cone-beam computed tomography. 
Sagittal sections from the CBCT revealed a close contact between the crown of the tooth number 3.3 and the roots of lower left incisors (Figure 3). Fortunately, there was no radicular damage concerning the permanent teeth. The axial sections helped us visualize the tooth projection in a mesio-distal direction and establish the appropriate coordinates for the surgical exposure (Figure 4, a-c).
There was no bone on the labial aspect of the alveolar process of the canine. Also, the presence of a follicular cyst was diagnosed, causing a malposition of the adjacent incisors. The canine was positioned bucally, and the lower left incisors were pushed to the lingual side, especially the tooth 3.2 . This tooth was also distally inclined.

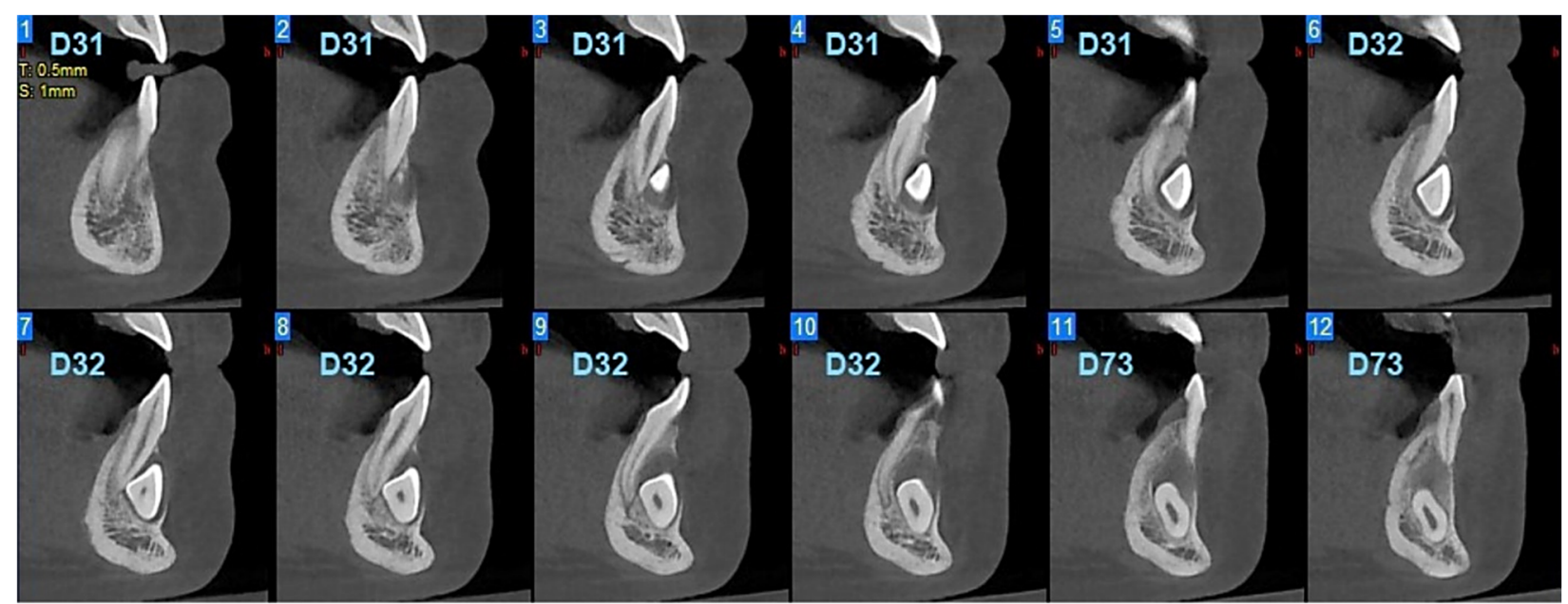

Figure 3 - Sections from the hemimandibular CBCT: the contact between the impacted canine and lower left permanent incisors. CBCT: Cone-beam computed tomography.
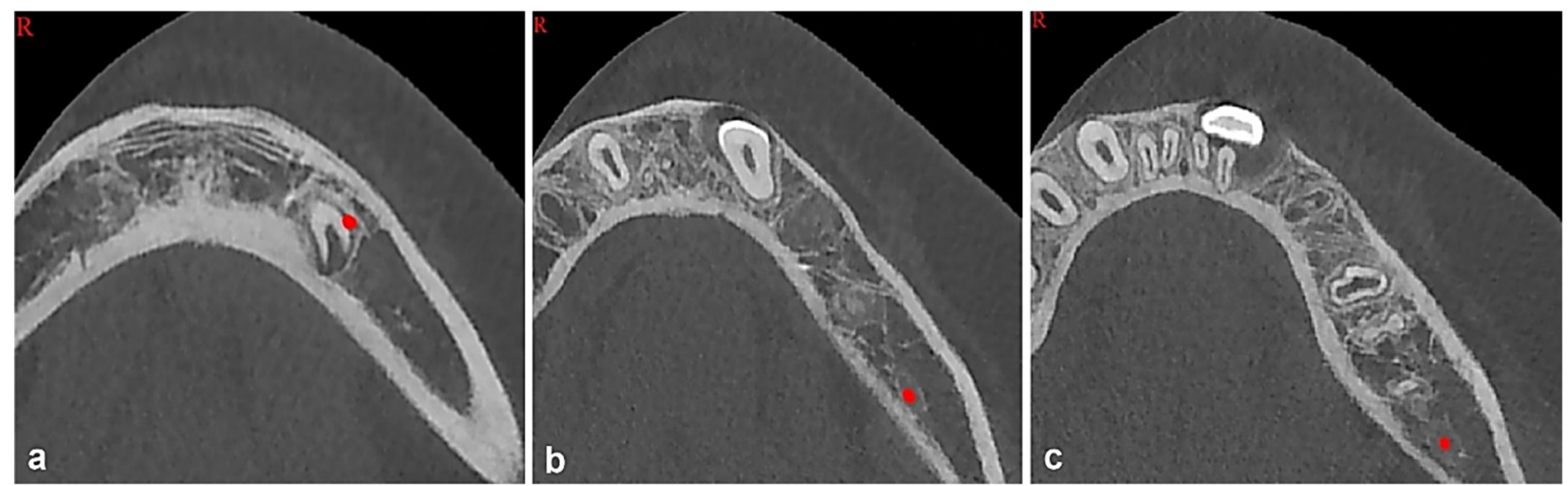

Figure $4-(a-c)$ The position of the impacted canine inside the mandibular bone.

Three-dimensional (3D) reconstructions are useful tools for a better visualization of the position of the impacted and transmigrating teeth, and also their relation with adjacent structures. They give an overview of the situation and help in the decision-making process. For example, a reconstruction is useful in cases with severe impactions in order to visualize the amount of hard tissues to be removed for exposure of the tooth or for its extraction, how big the osseous defect will be at the end, and if extra measures should be taken (marsupialization for big cysts, removal of other teeth, etc.). In our case, it helped us to establish that the initial forces must be directed distally to protect the anterior teeth (Figure 5, $a$ and $b)$.

Figure 5 - (a) Coronal view of the lower left hemimandible: $3 D$ reconstruction on $C B C T$; (b) A better visualization of the crown dimension and position of the tooth number 3.3. 3D: Three-dimensional; CBCT: Conebeam computed tomography; L: Left.
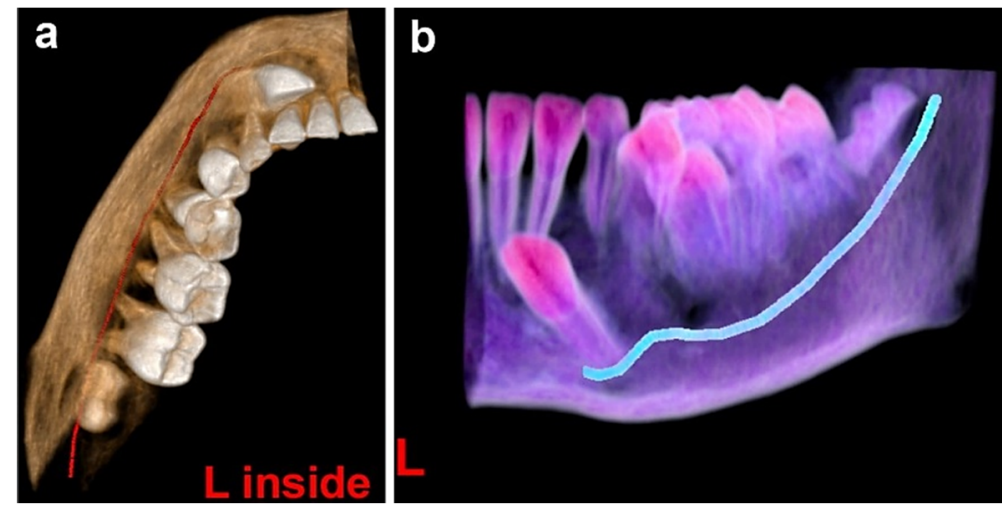
The treatment plan consisted of two phases: one in the mixed dentition and the other one in the permanent dentition. In the first phase, we have applied an upper removable appliance with an expansion screw, to increase the maxilla and maintain the transversal neutral relation between the upper and lower arches. In the mandible, it was important to provide a high anchorage for the traction of the impacted left canine, so we have used a lingual arch made from $0.9 \mathrm{~mm}$ stainless steel with bands on the first permanent molars and a soldered bar that connected the bands and was extending anteriorly, slightly contacting the lower incisors. Our idea to use a space maintainer appliance for anchorage in the mandible, like the lingual arch, is important because it ensures a simple and effective way to preserve the existing space, the transversal relation, and it permits a distal direction of the erupting force. It is also cheap, easy to manufacture, and can be designed and modified to best serve the clinical case.

The next step was the surgical one, represented by the removal of the left deciduous canine, the exposure of the labial aspect of the left permanent canine's crown, and the placement of a bonded attachment. We could see from the tomographic images that there was a thin layer of bone covering a part of the crown, so the tissues removed did not produce a significant defect. This is an important aspect, because we need a good amount of bone structure for the later positioning of the lower incisors. The attachment placed was a button with a chain and we considered that this was the safest accessory to use giving the important dental malposition and the big distance this tooth needed to travel (Figure 6, a and b). Also, the traction to the vestibular aspect of the band from the attachment was made using a stainless steel $0.12 \mathrm{~mm}$ ligature wire, so it was important to make advantage of the bigger diameter of the chain to prevent further impingement of the mucosa.
The initial connection with steel ligature was light, and the actual traction started after 14 days, with a combined rigid-elastic force, activating the elastomeric separator (Figure 7a). After five months, we took an additional record, a periapical radiography, to see the progress of the movement (Figure $7 \mathrm{~b}$ ). The canine was upright and closer to the occlusal plane. The premolars had a sufficient length of the roots, so we were able to begin the second phase of the treatment with fixed appliances.

To have an invisible appliance and raise our teenager's level of confidence, we opted for upper and lower aesthetic multibracket fixed appliance, with a 0.022 slot dimension and Roth prescription, also keeping the lingual arch. After levelling and aligning and after inserting a stainless steel archwire in the lower arch for good anchorage, we used an auxiliary 0.014 nickel-titanium (NiTi) wire. This wire helped us to move the canine in the occlusal direction and bring it in its normal position, without side effects on the adjacent teeth (Figure 8, a-f). It took us 20 months to bring the canine in its proper position. In the last picture, we can see the canine placed inside the dental arch and the treatment progression. Further orthodontic steps will follow and one of the most important is the finishing and detailing at the level of the migrating cuspid. Some stripping in the lower arch and additional lingual root torque at the level of tooth 3.3 will be required to position the root inside the bone and to have a better periodontal health and appearance. The aspect of the gingiva in the anterior region is good, thus meaning that we have a good level of supporting bone and that the surgical sutures were not tensed, allowing a normal healing rate.

We consider that, even at this stage, the case is a success because we were able to early diagnose a complex malposition and save a tooth that otherwise would have been extracted.

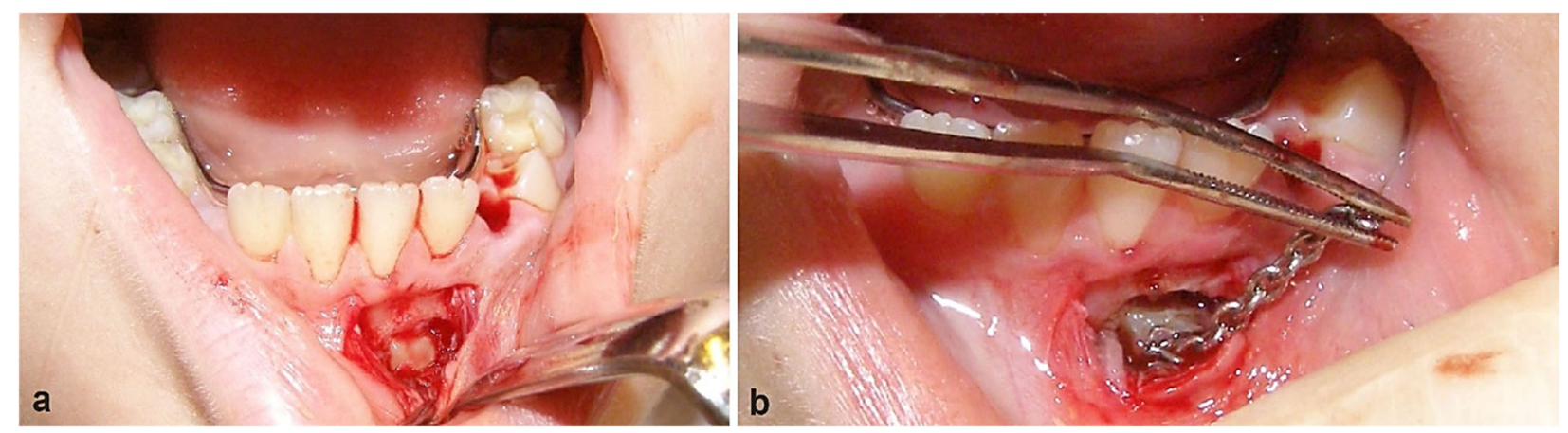

Figure 6 - (a and b) Surgical canine exposure and bonding of the attachment.

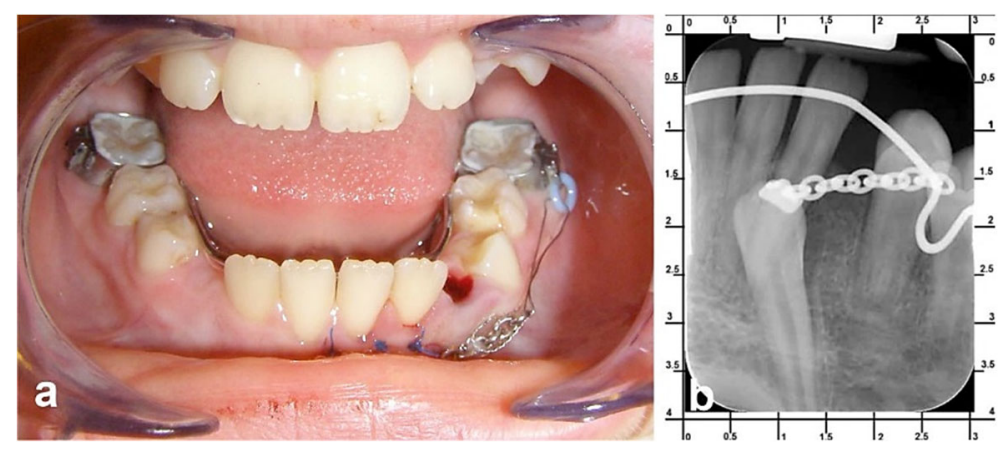

Figure 7 - (a) First phase of treatment combining lingual arch and exposure and traction of the impacted canine; (b) Radiological aspect regarding the progression of movement of the tooth number 3.3. 

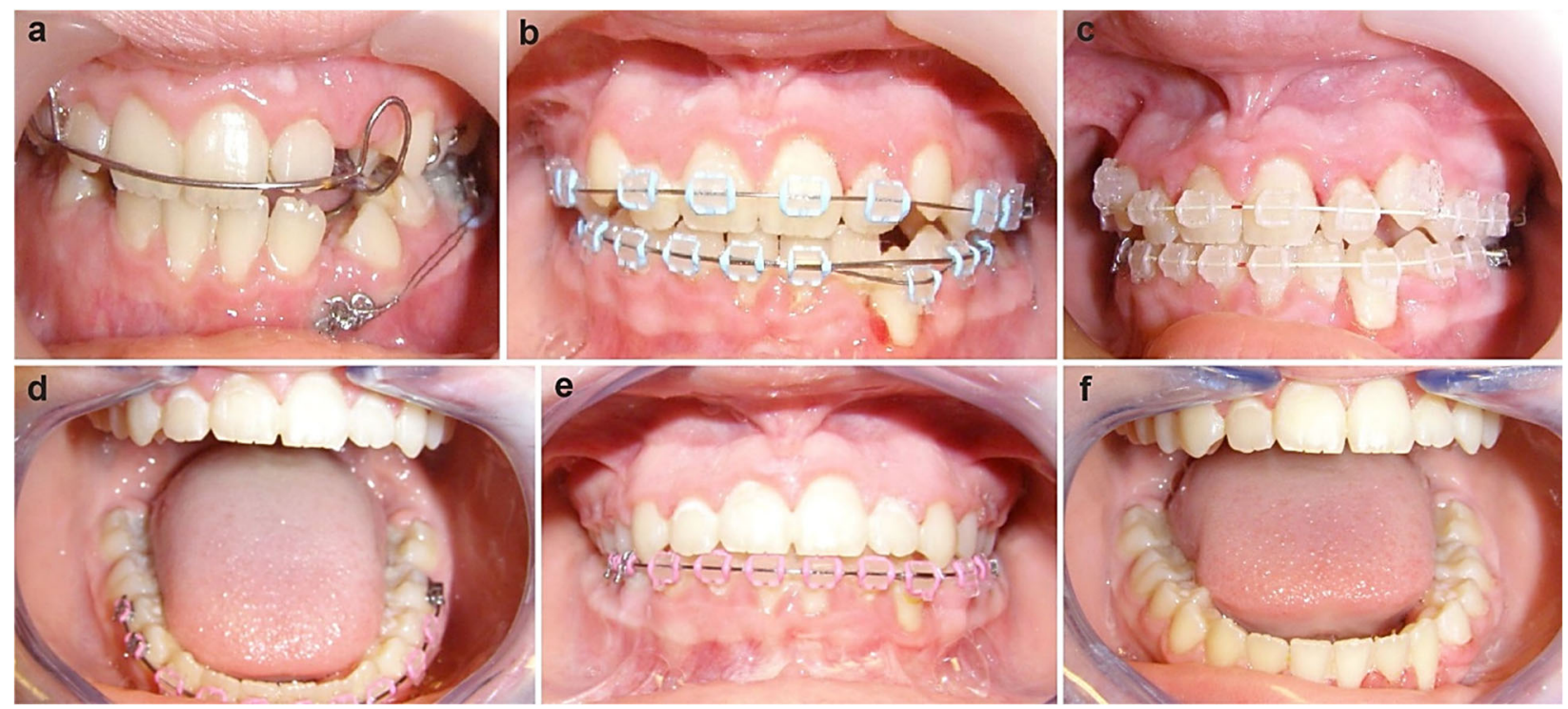

Figure 8 - "Recapturing” the malpositioned tooth: (a-c) treatment progression in various phases and (d-f) last phase of alignment to obtain a functional and esthetic result.

\section{口 Discussions}

Impacted mandibular canines are much less frequent than impacted maxillary canines, possibly because sometimes a more favorable order of eruption occurs in the lower arch (canine before the first premolar) [11,21], or because there is a bigger reserve given by the leeway space [22]. That can also be the reason why few articles and documentation exist about this aspect. In a study about the incidence of canine impaction and transmigration in a Turkish group, the impacted lower canines represented $0.46 \%$ and the transmigrated ones represented $0.34 \%$ [23]; our article not only reports one of these rare clinical situations, but also highlights the importance of early detection and of the multidisciplinary treatment steps that are important to take in these cases, to obtain a proper long-term result.

There are very few cases of successful orthodontic management of transmigrated canines reported [24]. Cases that have a more favorable prognosis and a good outcome are the ones diagnosed in the first stages of tooth migration $[7,8,13,16,25]$. Camilleri \& Scerri described a progressive alteration of the tooth position in four steps: in the first one the tooth development and position appear normal; in the second one, the tooth begins to deviate from its initial position; the third one describes the transmigration with mostly a mesial movement of the tooth and with maximum speed in the pubertal phase; finally, the tooth migrates only in apical and mesial direction and, combined with the osseous growth, the tooth stays in a low position [26]. The case that we presented was diagnosed between the second and third stage of this classification, so the early detection allowed a proper interdisciplinary orthodonticsurgical management, and a successful outcome.

Howard proposed a classification of transmigrated canines based on the angle between the long axis of the impacted tooth and the midsagittal plane. If the angle value is $25^{\circ}$ to $30^{\circ}$, the canine can change its position, but will not cross the midline; between $30^{\circ}$ and $95^{\circ}$, the tooth tends to cross the midline, with a more frequency when the angle value is over $50^{\circ}$ [9]; therefore, the axial inclination of the canine helps to predict the risk of impaction or transmigration. In the case presented above, the inclination of the impacted tooth 3.3 was $36^{\circ}$, so a clear tendency to transmigration could be detected.

The tools for diagnosis are both clinical and radiological. Buccal or lingual swelling, the persistence of the temporary canine, inclinations of lower incisors are aspects that can be detected at a periodical follow-up and indicate the probability of impacted, transposed, or transmigrated canine [23]. Additional radiographic investigations can be recorded, but the "gold standard" is represented by the $\mathrm{CBCT}$, which is the most accurate $3 \mathrm{D}$ diagnostic instrument. A thorough evaluation of the affected tooth position and its relation with other adjacent structures is possible on $\mathrm{CBCT}$, thus offering the best information for optimal treatment choices [27]. Bishara identified several treatment options for the maxillary impacted canines, which can be used also as a guide for other impacted teeth. The strategies vary from no treatment to extraction, to auto transplantation, prosthetic replacement, or surgical exposure and orthodontic traction, the last one being the recommended approach [28].

One of the big concerns with the transmigrated teeth is that the orthodontic mechanics is complicated. Even if the surgical exposure and bonding of an attachment might be performed, the clinician must move the tooth on a long distance, while keeping the roots of the other teeth intact and preserving the alveolar bone. Some authors suggests that horizontally impacted canines are harder to bring into the arch and sometimes these teeth should be extracted. Conversely, the buccally positioned lower canines, with a more favorable long axis, can be better managed by orthodontic treatment [29]. Stafne stated that the migration of a tooth usually occurs before the root development is completed. If the clinician can detect early the presence of malpositioned teeth, then the chance of successful orthodontic traction is higher [30]. This fact also has a strong significance in our case, due to the aesthetic and functional importance of the canines [31]. 
These cases necessitate a close attention from the medical team; if it is observed that the unerupted mandibular canine continues to incline mesially, an interceptive protocol should be followed, comprising extraction of the persistent deciduous canine and surgical exposure and orthodontic traction of the impacted cuspid [32]. For the presented case, our treatment plan included the extraction of the temporary lower left canine, surgical exposure, followed by traction of the permanent canine to a fixed appliance. It was important to guide the tooth in a distal direction, to relieve the anterior alveolar process and to obtain a proper positioning of the roots of the lower incisors. This therapeutic attitude agrees with Bishara's [28] recommendations to initially direct the orthodontic force to move the impacted tooth away from adjacent teeth, to prevent their damage [29]. In our case, the eruption was guided correctly and so the adequate position of the canine was successfully achieved.

\section{a Conclusions}

Transmigration of the mandibular canine is a rare anomaly of eruption and position; if it is diagnosed too late, accidentally after a radiological examination, the tooth can be compromised easily or, in any case, it can be very difficult to recover and realign into the arch. In the presented case, the fact that the problem was identified early, during the mixed dentition period when the canine was still in its original part of the mandible, just before crossing the midline, resulted in preserving the tooth, essentially with no damage to the adjacent structures. A proper monitoring attitude, a correct evaluation and a thorough clinical and imagistic examination of all our patients is important, to intervene as soon as possible. This case highlights that the early detection of mandibular canine transmigration assures better chances of bringing the tooth inside the dental arch in its assigned position and the importance of using a multidisciplinary orthodonticsurgical approach, to reestablish good function, esthetics, and occlusion.

\section{Conflict of interests}

The authors declare that they have no conflict of interests.

\section{Authors' contribution}

Cătălina Farcaşiu and Mirela Ileana Dinescu have equal contributions to this paper.

\section{References}

[1] van der Linden FP. Theoretical and practical aspects of crowding in the human dentition. J Am Dent Assoc, 1974, 89(1):139-153. https://doi.org/10.14219/jada.archive.1974.0308 PMID: 4525065

[2] Moshkelgosha V, Khosravifard N, Golkari A. Tooth eruption sequence and dental crowding: a case-control study. F1000Res, 2014, 3:122. https://doi.org/10.12688/f1000research.3196.1 PMID: 25110582 PMCID: PMC4111114

[3] Jain S, Debbarma S. Patterns and prevalence of canine anomalies in orthodontic patients. Med Pharm Rep, 2019, 92(1): 72-78. https://doi.org/10.15386/cjmed-907 PMID: 30957090 PMCID: PMC6448493

[4] Bahl R, Singla J, Gupta M, Malhotra A. Aberrantly placed impacted mandibular canine. Contemp Clin Dent, 2013, 4(2): 217-219. https://doi.org/10.4103/0976-237X.114850 PMID: 24015012 PMCID: PMC3757885
[5] Ongole R, Praveen BN (eds). Textbook of oral medicine, oral diagnosis and oral radiology. $2^{\text {nd }}$ edition, Elsevier, New Delhi, India, 2013, 11-61. https://www.worldcat.org/title/textbook-oforal-medicine-oral-diagnosis-and-oral-radiology/oclc/880900 208

[6] Tarsitano JJ, Wooten JW, Burditt JT. Transmigration of nonerupted mandibular canines: report of cases. J Am Dent Assoc, 1971, 82(6):1395-1397. https://doi.org/10.14219/jada. archive.1971.0216 PMID: 5280055

[7] Joshi MR. Transmigrant mandibular canines: a record of 28 cases and a retrospective review of the literature. Angle Orthod, 2001, 71(1):12-22. https://doi.org/10.1043/0003-32 19(2001)071<0012:TMCARO>2.0.CO;2 PMID: 11211293

[8] Auluck A, Nagpal A, Setty S, Pai KM, Sunny J. Transmigration of impacted mandibular canines - report of 4 cases. J Can Dent Assoc, 2006, 72(3):249-252. PMID: 16696892

[9] Howard RD. The anomalous mandibular canine. Br J Orthod, 1976, 3(2):117-121. https://doi.org/10.1179/bjo.3.2.117 PMID: 1065380

[10] Peck S. On the phenomenon of intraosseous migration of nonerupting teeth. Am J Orthod Dentofacial Orthop, 1998, 113(5):515-517. https://doi.org/10.1016/s0889-5406(98)702 62-8 PMID: 9598609

[11] Chaitanya P, Reddy JS, Suhasini K, Chandrika IH, Praveen D. Time and eruption sequence of permanent teeth in Hyderabad children: a descriptive cross-sectional study. Int J Clin Pediatr Dent, 2018, 11(4):330-337. https://doi.org/10.5005/jp-journals10005-1534 PMID: 30397378 PMCID: PMC6212657

[12] Celikoglu M, Kamak H, Oktay $\mathrm{H}$. Investigation of transmigrated and impacted maxillary and mandibular canine teeth in an orthodontic patient population. J Oral Maxillofac Surg, 2010, 68(5):1001-1006. https://doi.org/10.1016/j.joms.2009.09.006 PMID: 20188449

[13] Aydin U, Yilmaz HH, Yildirim D. Incidence of canine impaction and transmigration in a patient population. Dentomaxillofac Radiol, 2004, 33(3):164-169. https://doi.org/10.1259/dmfr/15 470658 PMID: 15371316

[14] Javid B. Transmigration of impacted mandibular cuspids. Int J Oral Surg, 1985, 14(6):547-549. https://doi.org/10.1016/s03 00-9785(85)80063-6 PMID: 3936805

[15] Joshi MR, Daruwala NR, Ahuja HC. Bilateral transmigration of mandibular canines. Br J Orthod, 1982, 9(1):57-58. https:// doi.org/10.1179/bjo.9.1.57 PMID: 6948576

[16] Anbiaee N, Akbarian G. Transmigration of impacted mandibular canines: a report of four cases. J Dent Mater Tech, 2013, 2(2):67-72. https://doi.org/10.22038/JDMT.2013.501

[17] Kuftinec MM, Shapira Y, Nahlieli O. A case report. Bilateral transmigration of impacted mandibular canines. J Am Dent Assoc, 1995, 126(7):1022-1024. https://doi.org/10.14219/jada. archive.1995.0279 PMID: 7629344

[18] Marks SC Jr, Schroeder HE. Tooth eruption: theories and facts. Anat Rec, 1996, 245(2):374-393. https://doi.org/10.1002/ (SICI)1097-0185(199606)245:2<374::AID-AR18>3.0.CO;2-M PMID: 8769674

[19] Greenberg SN, Orlian Al. Ectopic movement of an unerupted mandibular canine. J Am Dent Assoc, 1976, 93(1):125128. https://doi.org/10.14219/jada.archive.1976.0630 PMID: 1064653

[20] Pippi R, Kaitsas R. Mandibular canine transmigration: aethiopathogenetic aspects and six new reported cases. Oral Surg, 2008, 1(2):78-83. https://doi.org/10.1111/j.1752-248X.2008. 00014.x

[21] Kochhar R, Richardson A. The chronology and sequence of eruption of human permanent teeth in Northern Ireland. Int $\mathrm{J}$ Paediatr Dent, 1998, 8(4):243-252. https://doi.org/10.1046/j. 1365-263x.1998.00092.x PMID: 9927925

[22] Vyas MB, Hantodkar N. Resolving mandibular arch discrepancy through utilization of leeway space. Contemp Clin Dent, 2011, 2(2):115-118. https://doi.org/10.4103/0976-237X.83077 PMID: 21957388 PMCID: PMC3180833

[23] Aktan AM, Kara S, Akgünlü F, Malkoç $S$. The incidence of canine transmigration and tooth impaction in a Turkish subpopulation. Eur J Orthod, 2010, 32(5):575-581. https://doi.org/ 10.1093/ejo/cjp151 PMID: 20237077

[24] Wertz RA. Treatment of transmigrated mandibular canines. Am J Orthod Dentofacial Orthop, 1994, 106(4):419-427. https:// doi.org/10.1016/S0889-5406(94)70064-8 PMID: 7942658 
[25] Vaida L, Todor BI, Corega C, Băciuţ M, Băciuţ G. A rare case of canine anomaly - a possible algorithm for treating it. Rom J Morphol Embryol, 2014, 55(3 Suppl):1197-1202. PMID: 25607406

[26] Camilleri S, Scerri E. Transmigration of mandibular canines - a review of the literature and a report of five cases. Angle Orthod, 2003, 73(6):753-762. https://doi.org/10.1043/00033219(2003)073<0753:TOMCRO>2.0.CO;2 PMID: 14719743

[27] Farcaşiu C, Dinescu MI, Bătăiosu M, Căruntu A, Andrei OC, Tănăsescu LA, Scrieciu M, Dăguci L, Dăguci C. Using CBCT in analyzing position and treatment planning for maxillary impacted canines. dentalTarget, 2016, 11(4):10-13. https:// library.parker.edu/eds/detail $\mathrm{db}=\mathrm{d}=\mathrm{db} \& a n=120973783$

[28] Bishara SE. Impacted maxillary canines: a review. Am J Orthod Dentofacial Orthop, 1992, 101(2):159-171. https://doi.org/10. 1016/0889-5406(92)70008-X PMID: 1739070
[29] Beadnell SW. Chapter 16 - Management of the impacted canine. In: Bagheri SC, Bell RB, Khan HA (eds). Current therapy in oral and maxillofacial surgery. Elsevier-Saunders, St. Louis, Missouri, USA, 2012, 135-145. https://doi.org/10.1016/B9781-4160-2527-6.00016-5

[30] Stafne EC. Malposed mandibular canine. Oral Surg Oral Med Oral Pathol, 1963, 16:1330. https://doi.org/10.1016/0030-42 20(63)90408-0 PMID: 14082307

[31] Kumar S, Urala AS, Kamath AT, Jayaswal P, Valiathan A. Unusual intraosseous transmigration of impacted tooth. Imaging Sci Dent, 2012, 42(1):47-54. https://doi.org/10.5624/isd.2012. 42.1.47 PMID: 22474648 PMCID: PMC3314837

[32] Vichi M, Franchi L. [The transmigration of the permanent lower canine]. Minerva Stomatol, 1991, 40(9):579-589. PMID: 1758420

\section{Corresponding authors}

Luminiţa Dăguci, Associate Professor, DMD, PhD, Department of Prosthodontics, Faculty of Dentistry, University of Medicine and Pharmacy of Craiova, 2 Petru Rareş Street, 200349 Craiova, Romania; Phone +40720-047 004, e-mail: daguciluminita@yahoo.com

Constantin Dăguci, Associate Professor, DMD, PhD, Department of Prevention of Oro-Dental Diseases, Faculty of Dentistry, University of Medicine and Pharmacy of Craiova, 2 Petru Rareş Street, 200349 Craiova, Romania; Phone +40728-272 222, e-mail: dagucicristi@yahoo.com

Received: October 10, 2020

Accepted: November 28, 2021 